which our surveys were made is not constant but varied from $19^{\circ} \mathrm{E}$. in Cape Town to about $70^{\circ} \mathrm{E}$. in the position of $20^{\circ} \mathrm{S}$. geomagnetic latitude, some longitude effect should be included in the observed intensity-latitude curve. For example, double circles in Fig. I illustrate the mean neutron intensity in Cape Town, where the ship stayod for about a week every one passage. Each of thom largely deviates from the respeotive latitude curve $A A^{\prime}$, amount. ing to 5 per cent in average. Correction for this amount of longitude effect leads to upward shifting of tho position of the first 'knee' by only a few dogreos. Nevertheless, there should still remain some significant difference between the air-altitude 'kne日' and the sea-levol one. This also may be concerned with any primary spectrum around Capo Town anomaly. Detailed consideration and discussion of the foregoing probloms will bo published elsowhere. In this article wo wish to point out the fact that some anomalous effect in the primary cosmic-ray spectrum due to the strong geomagnetic anomaly is observable even at sea-level. In any ovent, it will be necessary to measure altitude and latitude dependences of the various components by balloon flight around Cape Town anomaly.

The cosmic.ray measuremont aboard was supported by the Seienee Council of Japan, as one of the Japanese $\Lambda$ tarctic Research Expedition programmes. I thank Dr. Y. Miyazaki for his advice.

\section{Masahiro Kodama}

The Instituto of Physical and Chernical Research, Tolkyo.

'Kodama, M., Sci. Papers Inst. Phys. Chem. Res., 54, 20 (1960). 'Kodama, M., Fukushima, S., and Makino, T., J. Phyr. Soe. (Japan), Supp.
A, 2, 441 (1962).

"Webber, W. R., and Quenby, J. J., Phil. Mag., 4, 654 (1959).

4Quenby, J. J., and Webber, W. R., Phil. Mag., 4, 90 (1959).

${ }^{5}$ Quenby, J. J., and Wenk, G. J., Phil. Mag., 7, 1457 (1962).

du Plooy, N. F., Kühn, G. J., Maree, J. 1'., Stoker, P. H., and van der Walt, A. .I., nreprint (1962).

\section{Breaking Extension of Nylon Filaments under Combined Axial and Transverse Stresses}

The normally aecepted coaxial helix model of the goometrical structure of a continuous filament textile $\operatorname{yarn}^{1}$ is such that rupture of the yarn would be expceted to commenco at its centro, where the filaments are aligned parallel with the yarn axis. Hearle and Thakur ${ }^{2}$ havo shown for short lengths of yarn extended at low strain rates that breakage of tho yarn begins at a point away from the yarn centre. They concluded that this type of rupture would be expected if the centre filaments of the yarn were buckled in the unstrained state. This explanation, however, ignores any alteration in filarnent breaking extension which may bo associated with tho transverse stresses exerted by the surrounding filaments. These stresses have been shown ${ }^{8,4}$ to modify considerably the stress distribution in rubber filaments which form a twistod structure of the type described here. Some rosults which I have obtained recently on the broaking extensions of nylon monofilaments subjocted to a combined axial stress and transverso comprossive stress show that their extensions at break are changed under these conditions.

Model yarns consisting of a straight centre filament of low breaking extension around which are wrapped five filaments of medium breaking oxtension in a helical configuration have been constructed. These models are designed to ensure that, on stretching, the centro filamont breaks first. Using an Instron machine, the models were stretched until the centre filaunent broke and the strain at this point was observed. This orrosponds to tho breaking strain of the centre filament when subjected to a tensile stress along its axis together with a transverse compressive stress. By varying the initial helix angle of the outor filaments, a range of values of breaking extensions and

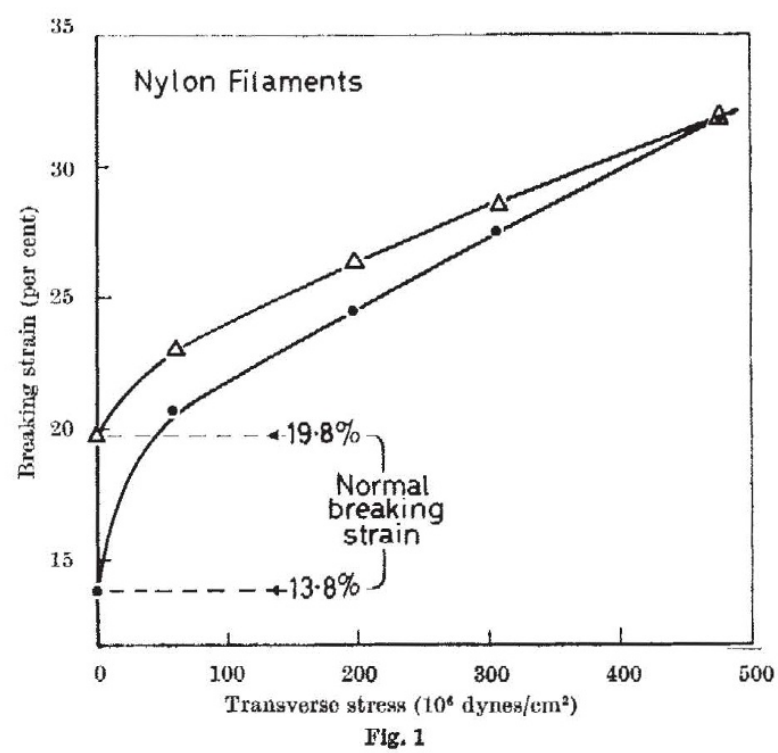

corresponding transverse stresses has been obtained. These stresses were calculatod from a knowledge of the yarn geometry at the breaking point and the stress-strain property of the outer filaments. Results given here show the relation between the breaking extensions and the calculsted stresses for two nylon filaments with normal breaking extensions of 13.8 and 19.8 per vent. It is seen that the strains at which the two specimons break increase considorably with transver'se stress, particularly for the specinen with the lower breaking extension. This effect has also boon observed in cellulose and 'Terylene' monofilaments. It should be noted at this point that an increase in breaking extension may not necessarily oceur when the transverse stresses aro applied via a fluid, for such a stress system would differ from that used here in that it would not be accompanied by large friotional forces acting over the filament surfaco. Whilo not rejocting the likely explanation of this effect as being the presence of the transverse stresses, it might be explained by tho frictional forees alone which bring about a more uniform distribution in stress along the filament.

Filaments at the centre of a yarn under tonsion are subjected to the largest transverso stresses so that their breaking extensions will be increased by a greater amount than those away from the centre, where the stresses are less. It follows from this that the precipitation of rupture of a textile yarn might begin at a point away from its axis even when the centre filaments are straight and parallel to the yarn axis.

An offoct of this kind might be an important factor which has previously beon neglected in investigating the mechanism of yarn rupture.

\section{Cotton silk and Man-made Fibres Resoarch Association, Shirley Institute, Didsbury, Manchester, 20.}

1 Platt, H. M., Test. Res. J., 20, 1 (1950).

${ }^{2}$ Hearle, J. W. S., and Thaknr, V. M., J. Text. Inst., 52, T, 49 (1061).

"Wilson, N., Bril. J. App. Phys., 13, 323 (1962).

4 Trcloar, L. R. G., Brit. I. App. Phys., 13, 314 (1962),

\section{ENGINEERING}

\section{Fluid Extrusion as a Drawing Process}

Fuvid extrusion is normally conducted as shown in Fig. 1a, where the diameter of the billet is smaller than the maximum diametor of the die opening. A high hydrostatic pressure forces the metal to flow through the die into a low hydrostatic pressure. 